\title{
A heuristic model of three-dimensional spectra of temperature inhomogeneities in the stably stratified atmosphere
}

\author{
A. S. Gurvich \\ A.M. Obukhov Institute of Atmospheric Physics, Russian Academy of Sciences, Moscow 109017, Russia \\ Received: 25 March 1996 / Revised: 14 November 1996 / Accepted: 3 December 1996
}

\begin{abstract}
Many measurements have shown that the random temperature fields in the stably stratified atmosphere are not locally isotropic (LI). The local axial symmetry (LAS) hypothesis looks more appropriate under these conditions. The object of this paper consists in the development of a flexible tool for spectral studies of LAS scalar fields independently of their origin in stably stratified geophysical flows. A heuristic model of a 3D spatial spectrum is proposed in order to describe and study statistical properties of LAS temperature inhomogeneities from LI structures up to quasi-layered ones. To simplify the solution of this problem, a main assumption was accepted: the consideration is restricted to $3 \mathrm{D}$ spectra which may be given on a one-parametric family of surfaces of rotation. Such 3D spectra may be represented by a single function of one variable which is the parameter of the family. This approach allows one to introduce the generalized energy spectrum which describes an energy distribution according to inhomogeneity sizes. The family of surfaces determines the shape of inhomogeneities. The family of ellipsoids of rotation and power-law generalized energy spectrum is used as the simplest example of the model application in order to study the general properties of LAS-structure spectra. The behavior of vertical, horizontal, and oblique 1D spectra and coherency spectra is studied. The relationship between the suggested model and some existing models of temperature spectra is considered. The application of the model for the analysis of experimental data is shown for two sets of measurements. It is shown that the suggested model may quantitatively describe experimental $1 \mathrm{D}$ spectra and coherency spectra from a unique point of view. It is noted that the model may be used for both the planning of measurements and data processing. Possible generalizations of the model are considered for random fields with more degenerate symmetry and for space-temporal spectra.
\end{abstract}

\section{Introduction}

The Obukhov-Corrsin model is employed most extensively at present in describing the spectra of temperature fluctuations in the turbulent atmosphere. Obukhov's theory of temperature fluctuations as a passive admixture is based on Kolmogorov's ideas of a statistically locally isotropic (LI) structure of random velocity field in a developed turbulent flow (Monin and Yaglom, 1975). Local isotrophy is the result of the assumption that all the directions in a certain domain of flow are physically equivalent. Consequently, the 3D field of temperature fluctuations is also statistically locally isotropic. However, under stable stratification, especially in the stratosphere or upper troposphere, there are no strong grounds for this assumption due to the effect of buoyancy forces on the evolution of inhomogeneities.

If the effect of buoyancy forces on motion is taken into account, the vertical direction becomes preferable. However, if we ignore the influence of the Earth's rotation, all directions in the horizontal plane remain equivalent. As is known, this is true for motions with scales less than $\left(\varepsilon / f_{c}^{3}\right)^{1 / 2}$, where $\varepsilon$ is the energy dissipation rate and $f_{c}$ is inertial frequency. Therefore, it is reasonable to assume that the random structure of the flow has the local axial symmetry (LAS) if this flow is stratified by density in the vertical direction and its boundaries are far away (Monin, 1988).

The results of measurements taken in both the stably stratified stratosphere and upper troposphere manifest anisotropy of inhomogeneities with different vertical scales. Let us mention just some of the results:

i. A dependence of clear-sky radar returns on the direction of sounding (Dalaudier et al., 1989; Gurvich and Kon, 1993), with a scale of some meters.

ii. Observations of extraterrestrial sources through the atmosphere from the spacecraft board (Gurvich 
et al., 1983; Alexandrov et al., 1990), with a scale of some tens of meters.

iii. A difference between the slopes of vertical and horizontal spectra (Hostetler and Gardner, 1994), with a scale of some hundred meters.

The kinematic theory of axisymmetric turbulence is considered for velocity field fluctuations (Chandrasekhar, 1950; Batchelor, 1953). Some properties of the LAS turbulence were considered (Sreenivasan and Narasimha 1978; Kristensen et al., 1983; George and Hussein, 1991), mainly for the case of small deviations in the velocity field properties from the LI. The properties of the spectral tensor were considered for a more degenerative case of symmetry of the boundarylayer velocity field when the symmetry exists both with respect to the horizontal plane and with respect to the vertical plane parallel to the direction of mean wind velocity (Kristensen et al., 1987).

The paper by Obukhov (1959) was one of the first where the effect of buoyancy forces on the structure function of small-scale temperature fluctuations in the atmosphere was taken into account. A possible typical horizontal scale was estimated defining the limit of applicability of the assumption of isotropy. The vertical scale of a similar kind was discussed in Lumley (1964) and Ozmidov (1965). The anisotropy of turbulence on the altitudes about of $90 \mathrm{~km}$ was considered in Dougherty (1961). Proceeding from the symmetry properties, some estimates were obtained in Monin (1965) for the single-point second moment of turbulent fields in the atmospheric boundary layer.

To take into account the deviations of temperature field from isotropy, the models are usually considered where the $3 \mathrm{D}$ spectrum of temperature fluctuations is assumed to be proportional to $\left[k_{z}^{2}+\eta_{0}^{2}\left(k_{x}^{2}+k_{y}^{2}\right)\right]^{-11 / 6}$ (Woo et al., 1980; Dalaudier et al., 1989). The coefficient $\eta_{0}=$ const. may be used as a measure of anisotropy; $\eta_{0}=1$ corresponds to the LI model. Such models are probably suitable for a consideration of spectra in some narrow region of the wave space. It is obvious that the anisotropy coefficient may change in considering a wide region of wave numbers.

The mentioned approaches used the Reynolds's idea of developed turbulence. It means that the main physical phenomenon is a random cascade breakdown of large dynamically unstable eddies with the subsequent creation of lesser unstable eddies continuing up to the creation of eddies so small that they disappear because of the influence of molecular viscosity. If the mean temperature gradient in such a turbulent flow exists, then random eddies generate the random temperature field.

However, alongside that already considered, the different source of temperature fluctuations may exist in a stably stratified atmosphere. This is a random set of inner gravity waves. Vertical displacements caused by wave motions provide temperature fluctuations. Garret and Munk (1975) considered the space-time energy distribution for an ensemble of random inner gravity waves inside the stably stratified ocean. This model was improved for atmospheric conditions (Van Zandt, 1982; Sidi et al., 1988). The models (Gardner et al., 1993; Gardner, 1994) are the slightly improved Garret-Munk model using the more complicated form of $m$-spectrum. This allows one to obtain the different slopes in vertical and horizontal $1 \mathrm{D}$ velocity spectra.

The initial Garret-Munk model corresponds to axisymmetric inhomogeneities. However, it and some of the following models contain the inertial frequency as a parameter. The latter is connected with the direction of the angular velocity of the Earth's rotation. Fritts and Van Zandt (1993) generalized the Garret-Munk model to take into account this second, preferable direction. Their generalization leads to a symmetry more degenerate than axial. The properties of this degenerate symmetry may well be important for large-scale inhomogeneities with scales of more than $\left(\varepsilon / f_{c}^{3}\right)^{1 / 2}$ or comparable with this horizontal scale.

An alternative approach was used in Dewan (1994). The author considered only 1D spectra and assumed on a semi-empirical basis that both vertical and horizontal 1D temperature spectra are the power law but with different slopes: -3 and $-5 / 3$, respectively. A summary of the power-law dependencies was published in Hostetler and Gardner (1994) for several models of 1D spectra of inner gravity waves.

It is obvious that the spatial random temperature field in the atmosphere is a 3D field and its second statistical moments (1D spectra for example) are determined by the properties of the $3 \mathrm{D}$ spectrum. The statistical approach to the description of these fields may be practically independent of its origin. The methods of the description have been very well developed for LI random fields (Monin and Yaglom, 1975). The situation is different with respect to LAS structure. Of course the Garret-Munk model and its generalizations are 3D spatial spectra of velocity fluctuations with strong anisotropic properties. However, these models are hardly connected with the linear theory of inner gravity waves through the polarization and dispersion equations. (The latter allows one to express the temporal spectrum using the spatial, but temporal spectra are not considered in the present paper). At the same time it is obvious that linear inner gravity waves are not the unique origin of temperature fluctuations.

The scintillation observations (Alexandrov et al., 1990) show definitely on the strong anisotropy of inhomogeneities with vertical scales of some tens of meters. Radar observations (Dalaudier et al., 1989; Gurvich and Kon, 1993) show the significant anisotropy of inhomogeneities with vertical scales of some meters. It is with only a small probability that such small scale anisotropic inhomogeneities are directly associated with linear inner gravity waves. However, there is no convenient model for theoretical studies, parameterization, and a description of such temperature fields. The object of this paper consists in the development of a flexible tool for the study of all spectral properties of non-LI spatial structures, independently of their origin.

The heuristic model of $3 \mathrm{D}$ spatial spectra is suggested in this paper for LAS inhomogeneities (LASIs) with 
variable anisotropy. This model is based on the fundamental symmetry properties. The main assumption is that the 3D spectra are given on a one-parameter family of surfaces of rotation in the wave-number space. The model is developed mainly for stably stratified geophysical flows as a generalization of the spectral theory of LI random fields. It will be used for the theoretical study of spectral properties of LASIs, for a parameterization, and a description of experimental data. This model may be applied to fluctuations in temperature or arbitrary scalar quantity; it may also be used for studies of spectral properties of LAS random fields from LI structures up to quasi-layered ones and/or up to structures strongly stretched along the symmetry axis. Some applications of the model are shown on the examples of measured vertical and horizontal 1D spectra and of measured coherency spectra. Some generalizations of the model are considered also for the more degenerate symmetry.

\section{Reduction of the 3D spectrum of LASIs to a function of one independent variable}

As follows from the routine definition of the LAS structure of random fields (Batchelor, 1953; Monin, 1988), the 3D spectrum $\Phi(\boldsymbol{k})$ of LASIs is a function of two variables: $\left|k_{z}\right|$ and $k_{h}=\left(k_{x}^{2}+k_{y}^{2}\right)^{1 / 2}, \quad \Phi(\boldsymbol{k})$ $=F\left(\left|k_{z}\right|, k_{h}\right)$, where $k_{x}, k_{y}, k_{z}$ are the components of the wave vector $\boldsymbol{k}, O z$ is the symmetry axis. Therefore, $\Phi(\boldsymbol{k})$ has constant value $\Phi^{(0)}$ on the surfaces of the rotation around the $k_{z}$-axis: $\Phi(\boldsymbol{k})=\Phi^{(0)}$.

Note that these surfaces are the family of concentric spheres for LI inhomogeneities. Each of these spheres is determined by one parameter: the radius $k$. In this case, the $3 \mathrm{D}$ spectrum is the function of this one parameter.

The given geometric interpretation prompts a possible way of separating a certain class of 3D spectra of LASIs in order to reduce them to a single function of one variable using the symmetry properties (Gurvich, 1995). This significantly simplifies the further development of the model. To take advantage of this we will assume that the considered 3D spectra take constant values on some one-parameter family of surfaces of rotation,

$G\left(\left|k_{z}\right|, k_{h} ; q\right)=0$,

in the 3D wave-number space. Let us assume that only one single-connected surface corresponds to every value of the parameter $q$ in Eq. (1) and all the surfaces of this family fill the wave space without crossing. Under these conditions, the $3 \mathrm{D}$ spectrum may be considered as the function $\phi(q)$ of only one variable $q$,

$F\left(\left|k_{z}\right|, k_{h}\right)=\phi(q)$,

instead of the function of two variables as in the general case. Equations (1) and (2) are the mathematical expression of the main assumption used in the creation of this heuristic model.

As is known, the energy spectrum $E(k)$ is often used together with the 3D spectrum to study LI random fields, and in this case it is defined as the integral of the 3D spectrum on angular variables in wave-number space. The relationship between $E(k)$ and $\Phi(\boldsymbol{k})$ was discussed in one of the first papers on the spectral theory of LI turbulence (Obukhov, 1941). Corresponding calculations give: $E(k)=4 \pi k^{2} \Phi(\boldsymbol{k})$. Such a definition is connected with the symmetry properties as $\Phi$ is given on the family of concentric spheres for LI random fields.

The assumption that $\Phi(\boldsymbol{k})$ has a constant value on the surfaces of a one-parameter family provides a reasonable way of introducing the concept of the generalized energy (GE) spectrum $E(q)$. It is natural to define the GE of LASIs in the following way - in connection with the accepted assumption -

$E(q) d q=\int_{d v_{q}} \Phi(\boldsymbol{k}) d^{3} \boldsymbol{k}$,

where $d v_{q}$ is the volume of wave space bounded by two surfaces of rotation [Eq. (1)] with the parameters $q$ and $q+d q$. Definition (3) coincides with the usual definition of $E$ for LI random fields.

As follows from Eq. (3), $E(q)$ is the energy density with respect to $q$. It is convenient to choose the dimension of $q$ in Eq. (1) to be the same as the wave number $k=|\boldsymbol{k}|$ for this reason. The final choice of the family given by Eq. (1) and its parameter $q$ is dictated by a priori information about the considered random field and conditions of simplicity of the model.

\section{Choice of family of surfaces for theoretical studies}

The preceding considerations of general symmetry properties show that the suitable choice of a family satisfying Eq. (1) is the main problem for the creation of spectral models of LASIs. An ellipsoid of rotation is the simplest surface which may satisfy the symmetry conditions. At the same time ellipsoids may reflect one of the main properties of LASIs: the ratio of vertical and horizontal typical scales. Thus the family of ellipsoids of rotation may be a convenient example for the theoretical study of some general spectral properties of LAS random fields. Let us therefore assume as a first step that the 3D spectrum $\Phi(\boldsymbol{k})$ has a constant value on the one-parameter family of ellipsoids of rotation about a vertical axis $k_{x}=k_{y}=0$ :

$G\left(\left|k_{z}\right|, k_{h} ; q\right)=k_{z}^{2}+\eta^{2}(q) k_{h}^{2}-q^{2}=0$,

where $q>1 / L_{0}$ is the semi-axis along $k_{z}$ and represents the parameter of the family. The ratio of semi-axes $\eta(q)$ is a function of $q, \eta \equiv 1$ corresponds to LI random fields. The outer scale $L_{0}$ may mean a maximum vertical scale for which an assumption of LAS is true. To make the introduced $\Phi$ an unambiguous function of $\boldsymbol{k}$, it is sufficient that $\eta(q)$ should be a continuous unambiguous differentiable function of $q$ and the condition

$\frac{q}{\eta(q)} \frac{d \eta(q)}{d q}<1$ 
should be fulfilled for all $q L_{0}>1$. This condition implies the absence of ellipsoid intersections in the family given by Eq. (4).

The stable stratification of the atmosphere prevents a development of inhomogeneities in the vertical direction. The case $\eta>1$ corresponds to stretched ellipsoids in the wave-number space or flattened inhomogeneities in the observation space. Therefore the family given by Eq. (4) at $\eta \geq 1$ may be used for the study of spectra at stable stratification. The large $q$ corresponds to smallscale inhomogeneities. It is difficult to expect considerable deviations from isotropy because of the effect of molecular viscosity and molecular heat conductivity on the smallest scales $k l_{K}>1$, where $l_{K}$ is Kolmogorov's scale. Consequently we should pay special attention to wave numbers $k<1 / l_{K}$ while modeling and should have $\eta(q) \rightarrow 1$ at $q l_{K} \gg 1$. If we suppose that $\eta$ tends to 1 for large $q$, so all space is filled with ellipsoids of this family. The plot of Fig. 1 gives an idea of the shape of $F\left(\left|k_{z}\right|, k_{h}\right)$ surface. It shows schematically the level line projections of the $F\left(\left|k_{z}\right|, k_{h}\right)$ surface on the coordinate plane $k_{z} O k_{h}$.

The choice of stretched ellipsoids of rotation in the wave-number space is determined by the fact that inhomogeneities in the stably stratified atmosphere are stretched along the Earth's surface. The case $\eta<1$ (flattened ellipsoids of rotation in the wave-number space) corresponds to inhomogeneities stretched in the vertical direction. This may be possible, for example, for convection conditions in the atmosphere. The case $\eta \ll 1$ may allow us to study inhomogeneities for which the vertical correlation scale is much more than the horizontal one. A more detailed analysis of stretched LASIs is the subject of a separate investigation.

It is quite evident that the choice of ellipsoids given by Eq. (4) as a family of surfaces defining the model is not specific. This is necessitated by the simplicity of further analysis and the possibility of a simple limiting transition to the LI model when $\eta$ tends to 1 . At the same time the family of ellipsoids may represent quantitatively the inhomogeneity deformation along the symmetry axis. This is one of the main properties of non-spherical inhomogeneities. On the other hand, the possibility of choosing the functions $\eta(q)$ and $E(q)$ provides us with great potential for modeling.

The level lines for more complicated surfaces is shown in Fig. 1 for the family:

$$
\begin{aligned}
& k_{z}^{2}+\eta^{2}(q) k_{1}^{2}=q^{2}\left[1-\left(1-\eta^{-2}(q)\right) \cos ^{4}(2 \vartheta)\right]^{2}, \\
& \sin ^{2} \vartheta=\frac{k_{z}^{2}}{k_{z}^{2}+k_{1}^{2}} .
\end{aligned}
$$

This family may be used for example at $\eta \gg 1$ for the modeling of such a spectrum $F$, which, as a function of $\vartheta$ at fixed $k$, has deep minimums at $\vartheta=0, \pi / 2$, and $\pi$, like the Garret-Munk spectrum. At the same time Eq. (6) allows us to study the transfer to locally isotropic fields at large $q$ when $\eta$ tends to 1 . Note that the temperature spectrum corresponding to the Garret-Munk model may be represented exactly using the corresponding function $G$.
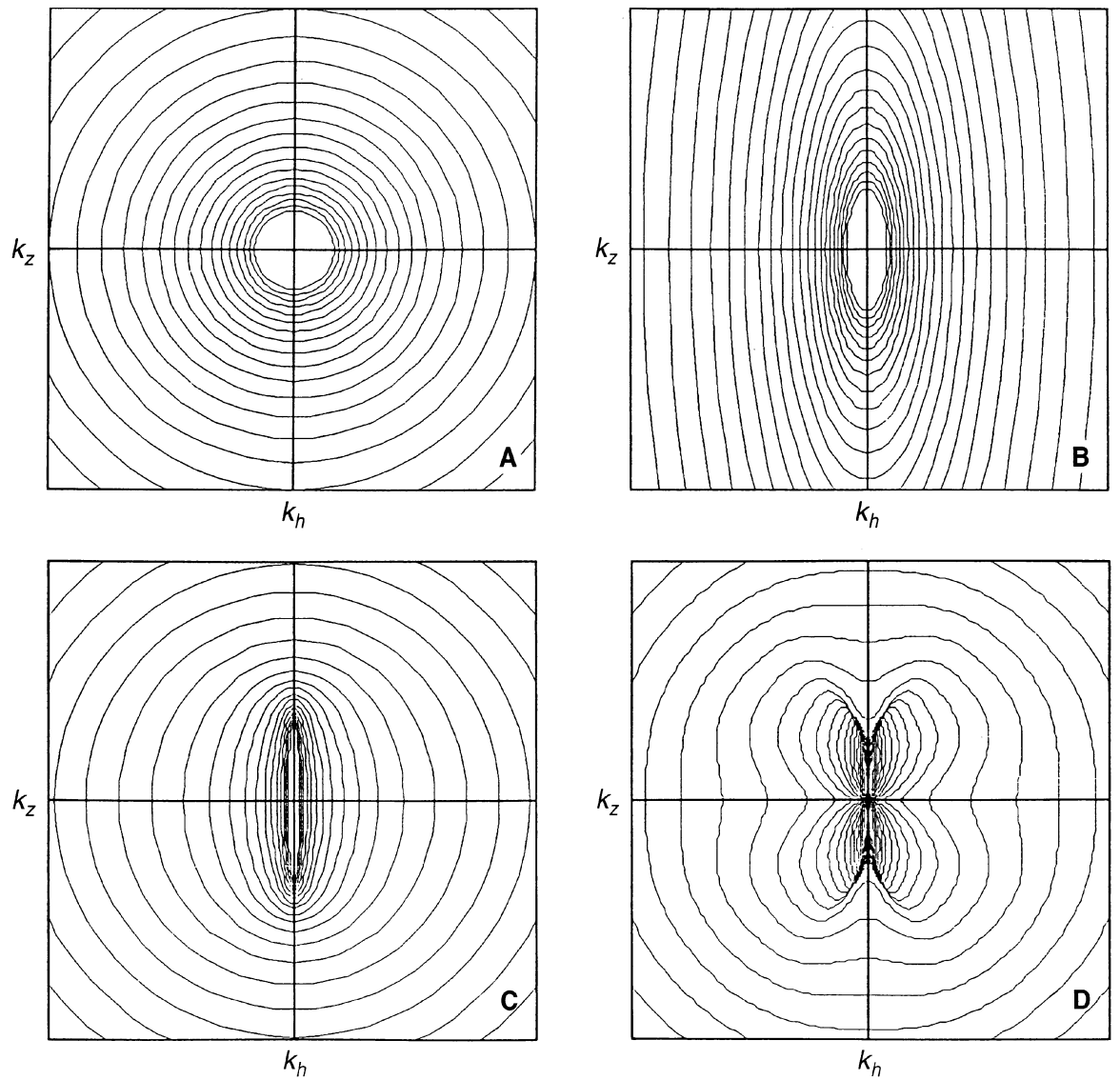

Fig. 1A-D. The scheme of the level line projections of a $F\left(k_{z}, k_{h}\right)$ surface on the coordinate plane $k_{h} O k_{z}$. A LI inhomogeneities, B LASIs with a constant anisotropy $\eta_{0}=3$, C LASIs with variable anisotropy, $\eta=1+q^{-6}, E(q)=q^{-3}$, and D LASIs that correspond to the family of Eq. (6), $\eta(q)$ and $E(q)$ are the same as for $\mathbf{C}$ 


\section{The physical meaning of the GE spectrum and anisotropy coefficient of LASIs}

The connection between the GE spectrum $E(q)$ and the $3 \mathrm{D}$ spectrum may be derived in the following way. The volume of the ellipsoid of Eq. (4) is equal to $v_{q}=4 \pi q^{3} / 3 \eta^{2}(q)$; consequently the volume between the surface of two ellipsoids of Eq. (4) with the semiaxes $q$ and $q+d q$ is equal to $d v_{q}=4 \pi q^{2} \eta^{-2}$ $[1-(2 / 3)(q / \eta)(d \eta / d q)] d q$. If we now use Eqs. (2) and (3) then

$E(q)=\frac{4 \pi q^{2}}{\eta^{2}(q)} \phi(q)\left(1-\frac{2 q}{3 \eta(q)} \frac{d \eta(q)}{d q}\right)$.

To make clear the meaning of the GE spectra $E(q)$ and $\eta(q)$ for LASIs, let us consider an example of the spectrum $\Phi(\boldsymbol{k})$ which is equal to zero everywhere except the surface of the ellipsoid Eq. (4) with $q=$ const. $=q_{1}$. Such a spectrum corresponds to a packet of plane waves $A \exp (i \boldsymbol{k} \boldsymbol{r})$ with random amplitudes $A$ and with wave vectors $\boldsymbol{k}$ whose ends lie on this surface. Having designated the total energy of inhomogeneities forming the packet as:

$E_{0}=\int \Phi(\boldsymbol{k}) d^{3} \boldsymbol{k}$

we can write an expression for their 3D spectrum:

$\Phi(\boldsymbol{k})=E_{0} \delta\left(\left(k_{z}^{2}+\eta^{2}(q)\left(k_{x}^{2}+k_{y}^{2}\right)\right)^{1 / 2}-q_{1}\right) \frac{d q}{d v_{q}}$,

where $q$ is connected with $\boldsymbol{k}$ by Eq. (4), $\delta$ is the delta function. The GE spectrum $E(q)$ is equal to:

$E(q)=E_{0} \delta\left(q-q_{1}\right)$

for the 3D spectrum of Eq. (9).

Having calculated the correlation function $B(\boldsymbol{r})$

$$
\begin{aligned}
B(\mathbf{r}) & =\int \Phi(\boldsymbol{k}) \exp (i \boldsymbol{k r}) d^{3} \boldsymbol{k} \\
& =E_{0} \frac{\sin \left\{\left[q_{1}^{2}\left(z^{2}+\left(y^{2}+x^{2}\right) / \eta^{2}\left(q_{1}\right)\right)\right]^{1 / 2}\right\}}{\left[q_{1}^{2}\left(z^{2}+\left(y^{2}+x^{2}\right) / \eta^{2}\left(q_{1}\right)\right)\right]^{1 / 2}}
\end{aligned}
$$

for Eq. (9), we can see that this wave packet forms LASIs with the vertical correlation scale $\pi / q_{1}$ and with the scale $\pi \eta\left(q_{1}\right) / q_{1}$ in the horizontal plane. Consequent$1 y$, it is reasonable to consider the ratio of these scales $\eta\left(q_{1}\right)$ as the anisotropy coefficient of LASIs with the typical vertical scale $\pi / q_{1}$. It is important that the anisotropy coefficient defined in such a way is determined for the 3D spectrum of Eqs. (2) and (4) for any point of wave space where $\Phi(\boldsymbol{k})$ exists.

The considered example of the $3 \mathrm{D}$ spectrum of Eq. (9) demonstrates the difference between the GE spectrum $E(q)$ and the $3 \mathrm{D}$ spectrum $\Phi(\boldsymbol{k})$. The latter describes the spectral density of the energy of plane waves $\exp (\boldsymbol{i k r})$ by which the expansion in spectrum is carried out, while $E$ is a spectral density of inhomogeneity energy. The use of $E(q)$ and $\eta(q)$ allows us to consider separately the energy of inhomogeneities and properties of their shape. It should be noted here that the meaning of $E(q)$ remains for more complicated families than Eq. (4), such as, for example, Eq. (6). However the exact meaning of $\eta(q)$ needs some specification in this case.

The non-dimensional anisotropy coefficient $\eta$ should be a function of the non-dimensional argument $\eta=\eta\left(q l_{s}\right)$, where $l_{s}$ is some scale. Proceeding from the assumption that anisotropy is more typical for largescale inhomogeneities and disappears for small-scale ones, we will assume that $\eta\left(q l_{s}\right) \rightarrow 1$ at $q l_{s} \gg 1$. We consider $l_{s} \ll L_{0}$ as a typical vertical scale of the symmetry change. As a prior estimation of $l_{s}$ the scale $l_{\mathrm{B}}=\left(\varepsilon / N^{3}\right)^{1 / 2}$ may be used (Lumley 1964; Ozmidov, 1965), where $N$ is the Brunt-Väisälä frequency. It should be noted here that $l_{s}$ can be considered in a definite sense as an outer scale of LI inhomogeneities.

Thus, the main assumptions Eqs. (1), (2) and the use of the family of Eq. (4) allow one to use two functions of one variable, i.e. the GE spectrum $E(q)$ and the anisotropy coefficient $\eta(q)$, for the representation of the 3D spectrum of LASIs, which is one function of the two independent variables $k_{z}$ and $k_{h}=\left(k_{x}^{2}+k_{y}^{2}\right)^{1 / 2}$. The GE spectrum describes the energy distribution while the anisotropy coefficient describes the family of surfaces on which the 3D spectrum is given. The use of two functions provides the necessary flexibility of the model, which is convenient for the theoretical study of spectral properties of LASIs.

However, the family of Eq. (4) is an example of Eq. (1) only, it is not the result of a solution of a problem of statistical fluid mechanics. Therefore, to determine the concrete form of $E(q)$ and $\eta(q)$, it is necessary to use various hypotheses, qualitative physical assumptions, dimension analysis, etc. Such approaches however are generally accepted in the study of turbulence. The application of the conceptions of $E$ and suitable family $G$ may somewhat simplify the use of these approaches. To verify the hypotheses nothing remains but to use the results of measurements.

\section{$51 D$ spectra of LASIs}

Direct atmospheric measurement of $\Phi(\boldsymbol{k})$ are available using a radar return technique (Tatarskii, 1967; Doviac and Zrnich, 1984). Such studies represent a quite difficult problem in a wide range of $\boldsymbol{k}$ change. The problem with the direct measurement of $E(q)$ is ever more difficult. Usually the experiment provides more easily measurable 1D spectra $V\left(k_{1} ; \vartheta\right)$ along some direction $\boldsymbol{n}$

$$
V\left(k_{1} ; \vartheta\right)=\int \Phi(\boldsymbol{k}) \delta\left(\boldsymbol{k n}-k_{1}\right) d^{3} k, \quad-\infty<k_{1}<+\infty
$$

where $\boldsymbol{n}$ is a unit vector, $\boldsymbol{n}=\{\cos \varphi \cdot \sin \vartheta$, $\sin \varphi \cdot \sin \vartheta, \cos \vartheta\}, \vartheta$ is the angle between $\boldsymbol{n}$ and the axis of symmetry, $\varphi$ is azimuthal angle $-\pi<\varphi<\pi$. We obtain for any horizontal direction $\vartheta=\pi / 2$ : 


$$
V\left(k_{1} ; \pi / 2\right)=\int \Phi(\boldsymbol{k}) d k_{y} d k_{z}, \boldsymbol{k}=\left\{k_{1} \cos \varphi, k_{1} \sin \varphi, k_{z}\right\} .
$$

For the vertical direction $\vartheta=0$ :

$V\left(k_{1} ; 0\right)=\int \Phi(\boldsymbol{k}) d k_{x} d k_{y}, \quad k_{1}=k_{z}$.

For the vertical spectrum we obtain from Eqs. (4) and (14) after changing the variables $k_{x}=æ \cos \varphi$ and $k_{y}=æ \sin \varphi$, where $æ^{2}=\left(q^{2}-k_{z}^{2}\right) / \eta^{2}(q)$, the following expression:

$V\left(k_{z}, 0\right)=\frac{1}{2} \int_{\left|k_{z}\right|}^{\infty} \frac{d q}{q} E(q) \frac{1-\frac{q^{2}-k_{z}^{2}}{q \eta(q)} \frac{d \eta(q)}{d q}}{1-\frac{2}{3} \frac{q}{\eta(q)} \frac{d \eta(q)}{d q}}$.

It is seen from Eq. (15) that for $\eta=$ const., the $1 \mathrm{D}$ vertical spectrum of LASIs does not depend on the anisotropy coefficient.

For the horizontal spectrum of Eq. (13) we obtain similarly a somewhat more complicated expression:

$V\left(k_{1}, \pi / 2\right)=\frac{1}{2} \int_{q_{m}}^{\infty} \frac{d q}{q} \eta(q) E(q) \frac{1-\frac{q}{2 \eta(q)} \frac{d \eta(q)}{d q}\left(1+\frac{k_{1}^{2} \eta^{2}(q)}{q^{2}}\right)}{1-\frac{2}{3} \frac{q}{\eta(q)} \frac{d \eta(q)}{d q}}$,

where $q_{m}$ is the root of equation $k_{1}=q_{m} / \eta\left(q_{m}\right)$. For $\eta=\eta_{0}=$ const. we obtain from Eq. (16) a simple expression:

$V\left(k_{1}, \pi / 2\right)=\frac{\eta_{0}}{2} \int_{\eta_{0} k_{1}}^{\infty} \frac{d q}{q} E(q)$.

It should be noted that Eqs. (15) and (16) may be used in the general case as the equations whose solution defines the functions $E(q)$ and $\eta(q)$ if both 1D spectra are known from the measurements. The solution should satisfy the conditions of Eq. (5) for $\eta$ and $E(q)$ should be positive. In the converse case we may conclude that the considered field cannot be represented using 3D spectra given on the family of Eq. (4).

\section{Power-law GE spectrum and anisotropy coefficient}

The power-law spectra are a typical topic of study in turbulence theory. Numerous experimental spectra are described also using power-law functions. If we assume that temperature fluctuations are determined by the following parameters of medium: $g / T$ (buoyancy parameter) and $N$ (the Brunt-Väisälä frequency) (Shur, 1962; Lumley, 1964) then we can write for the GE spectrum the following equation $E(q)=$ const. $\cdot(g / T)^{-2}$ $N^{4} q^{-3}$. If we assume that the rate of dissipation of kinetic energy of turbulence $\varepsilon$ and the rate of dissipation of temperature inhomogeneities $\varepsilon_{\mathrm{T}}$ are the determined parameters (Obukhov, 1949), then we can write for the GE spectrum the following equation: $E(q)=$ const. $\cdot \varepsilon_{\mathrm{T}}$ $\varepsilon^{-1 / 3} q^{-5 / 3}$. Therefore we shall take a close look at the power-law spectrum
$E(q)=S_{T}^{2} q^{-\mu+2}$.

As a first step we will also consider the case when the anisotropy coefficient $\eta$ is the power-law function of $q l_{s}$ :

$\eta(q)=1+\left(q l_{s}\right)^{-v}, \quad v>0$.

The numerical parameter $v$ characterizes the relative rate of anisotropy change at small $q l_{s} \ll 1$ as $(q / n)(d \eta / d q)=-v /\left(1+q l_{s}\right)$. The choice of Eq. (19) is caused by the condition of simplicity. Besides this the combination of Eqs. (18) and (19) may give spectral functions with a power-law asymptote which are often used in the observation approximations. We may see that such a choice provides the possibility of studying the transition from LI inhomogeneities $\left(q l_{s} \gg 1\right)$ up to layer structures as $q l_{s}$ tends to zero.

It is clear that $S_{T}^{2}$ is an analog of the structure characteristic of the LI spectrum. At $\mu=11 / 3$ and $\eta=1$ we could obtain $S_{T}^{2}=\left[10 /(9 \Gamma(1 / 3)] C_{T}^{2} \approx .4 C_{T}^{2}\right.$ where $C_{T}^{2}$ is the structure characteristic of the Obukhov-Corrsin model and $\Gamma$ is the gamma function. However, we introduce $S_{T}^{2}$ using the spectral representation because it is more convenient for exponents $\mu \geq 5$.

Figure 2 shows the deformation of the shape of the 3D spectra $F$ when the relative rate of the anisotropy coefficient change increases. All calculations of $F$ were made using Eqs. (4) and (18) at $\mu=5, S_{T}^{2}=1$. The anisotropy was calculated using Eq. (19) and $v=0.5$, $1,2.5$, and 5. The upper plot corresponds to $F\left(\left|k_{z}\right|, 0\right)$ the lower to $F\left(0, k_{h}\right)$. The anisotropy coefficients $\eta$ are shown as functions of $k_{z}$ and $k_{h}$. The very large values of $\eta$ correspond to a transition of quasi-layered random media for which horizontal correlation scales are many times more than the vertical. The numbers on the curves correspond to the values of $v$. The spectrum of LI inhomogeneities $(\eta=1)$ is shown by dashed lines for comparison. The data in Fig. 2 are connected with the special law Eq. (19) of an increase in the anisotropy with an increase in inhomogeneity scales. It is obvious however that similar behavior $F$ will be observed for $E(q)$ from Eq. (18) when $\eta(q)$ is a monotone decreasing function: $d \eta(q) / d q<0$.

We now consider the vertical spectrum Eq. (15) for $E$ from Eq. (18) and for $\eta$ from Eq. (19). From it can be obtained the simple asymptotic expressions for $V\left(k_{z}, 0\right)$ at $k_{z} l_{s} \gg 1$ :

$V\left(k_{z}, 0\right)=\frac{S_{T}^{2}}{2(\mu-2)}\left|k_{z}\right|^{-\mu+2}, \quad \eta=1, \quad k_{z} l_{s} \gg 1$.

We may assume at $l_{s} / L_{0} \ll k_{z} l_{s} \ll 1$ that $\eta(q)$ $\cong\left(q l_{s}\right)^{-v}$ and then

$V\left(k_{z}, 0\right)=\frac{(\mu+2 v)}{\mu\left(1+\frac{2 v}{3}\right)} \frac{S_{T}^{2}}{2(\mu-2)}\left|k_{z}\right|^{-\mu+2}$,

$l_{s} / L_{0} \ll k_{z} l_{s} \ll 1$.

It is obvious that Eq. (20) corresponds to the LI model: $\eta=1$. However the asymptote Eq. (21) is close to the LI model too because the factor $(\mu+2 v) /$ $[\mu(1+2 v / 3)]$ is roughly of order one at practically all $\mu$ and $v$ of interest. To see better the difference between 


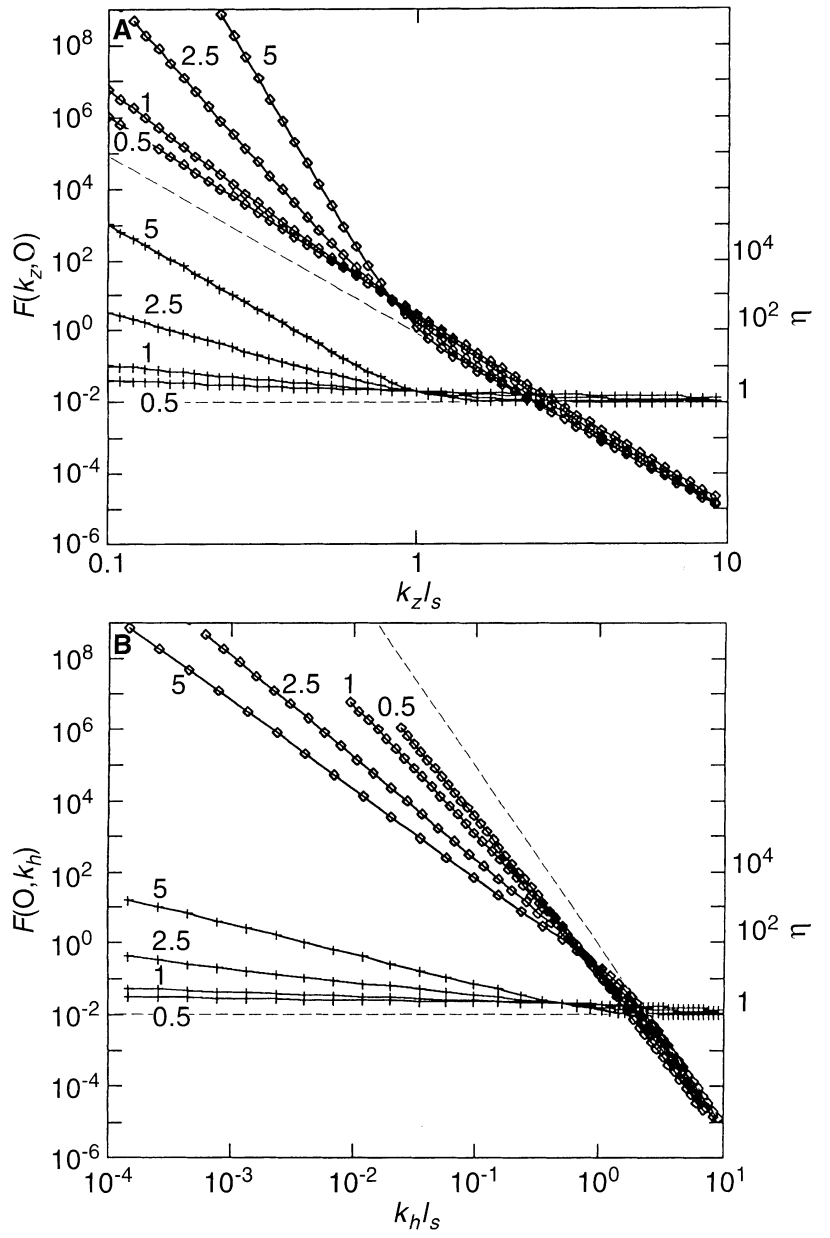

Fig. 2A, B. The 3D spectra $F\left(k_{z}, k_{h}\right)$ (diamonds) and anisotropy coefficients $\eta$ (crosses) for the family of Eq. (4) and for $E(q)=q^{-3}$ and $\eta(q)=1+\left(q l_{s}\right)^{-v}$. A $F\left(k_{z}, 0\right)$, $\mathbf{B} F\left(0, k_{h}\right)$; the numbers in the plots are the values of $v$; LI spectra and $\eta=1$ : dashed lines

the vertical spectra $V\left(k_{z}, 0\right)$ of LASIs and vertical spectra $V_{i s}\left(k_{z}\right)$ of LI inhomogeneities, the former were normalized to the latter. Such normalized spectra are shown in Fig. 3 as functions of $k_{z} l_{s}$. The anisotropy coefficient $\eta\left(q=k_{z}\right)$ is shown in this plot too. All calculations of $V\left(k_{z}, 0\right)$ were made using Eqs. (15) and (18) at $\mu=5,1 / L_{0}=0$. The anisotropy coefficient was calculated using Eq. (19) at $v=0.5,1,2.5$, and 5. This plot shows that vertical spectra of LASIs are close to those of LI inhomogeneities and the spectra $V\left(k_{z}, 0\right)$ slightly depend on the values of anisotropy. Hence the measurements of $V\left(k_{z}, 0\right)$ are useful for estimations of parameters $\mu$ and $S_{T}^{2}$ which determine the GE spectrum $E(q)$.

The horizontal spectrum $V\left(k_{1}, \pi / 2\right)$ Eq. (16) for Eqs. (18) and (19) has the high-frequency power-law asymptote

$V\left(k_{1}, \pi / 2\right)=\frac{S_{T}^{2}}{2(\mu-2)}\left|k_{1}\right|^{-\mu+2} \quad \eta k_{1} l_{s} \gg 1$

which of course coincides with $V_{i s}\left(k_{1}\right)$. The low-frequency asymptote

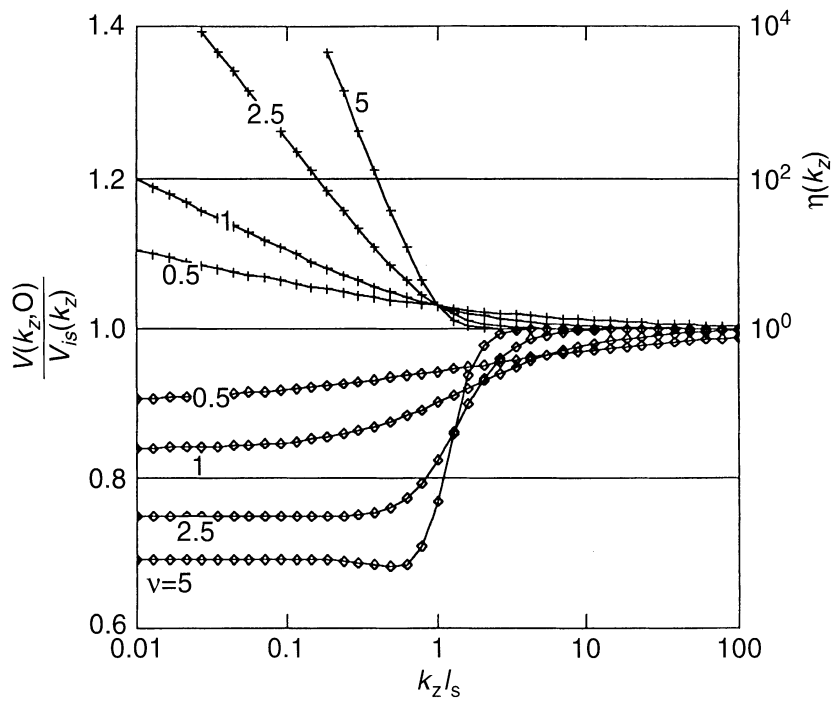

Fig. 3. The 1D vertical spectra $V\left(k_{z}, 0\right)$ of LASIs normalized to the 1D spectrum of LI inhomogeneities $V_{i s}\left(k_{z}\right)$ with the same GE spectrum $E(q)=q^{-3}$ (diamonds); anisotropy coefficients $\eta=1$ $+\left(q l_{z}\right)^{-v}$ (crosses), $q=k_{z}$; the numbers in the plots are the values of $v$.

$V\left(k_{1}, \pi / 2\right)=\frac{S_{T}^{2} l_{s}^{\mu-2}}{2\left(1+\frac{2 v}{3}\right)}\left(\frac{1+v / 2}{\mu+v-2}+\frac{v}{2(\mu+3 v)}\right)\left(k_{1} l_{s}\right)^{-\frac{\mu-2+v}{v+1}}$

may be obtained from Eq. (16) at $\eta k_{1} l_{s} \ll 1$ if we take into account that at finite value of $v$ and $\eta k_{1} l_{s} \ll 1$ we may assume that in Eq. (16) $\eta(q) \cong\left(q l_{s}\right)^{-v}, q_{m}\left(q_{m} l_{s}\right)^{v}$ $\cong k_{1}$ and $(q / \eta)(d \eta / d q) \cong-v$. It is clear from Eq. (23) that the low-frequency asymptote of the 1D horizontal spectrum $V\left(k_{1}, \pi / 2\right)$ is also the power-law function but with the different exponent $-(\mu-2+v) /(v+1)$. It is curious to note, in particular, that $(\mu-2+v) /(v+1)$ $=5 / 3$ if $\mu=5$ and $v=2$.

Figure 4 shows calculated horizontal spectra $V\left(k_{1}, \pi / 2\right)$ as functions of $k_{1} l_{s}$ for $\mu=5,1 / L_{0}=0$ and $S_{T}^{2}=1$. The anisotropy coefficients $\eta$ are also shown in this plot as functions of the same argument. The values of $v$ in Eq. (19) at calculations were equal to $v=0.5,1,2.5,5$. The dashed line corresponds to the LI model $\eta \equiv 1$. The numbers near the curves are the values of $v$. The power-law asymptotes Eqs. (22) and (23) are seen very clearly. The behavior of the horizontal spectrum $V\left(k_{1}, \pi / 2\right)$ in an intermediate domain $\left|\eta k_{1}\right| \simeq\left(l_{s}\right)^{-1}$ depends on the rate of the anisotropy coefficient change.

The plot shows that measurements of the 1D horizontal spectra are sensitive to anisotropy changes. It shows in accordance with Eq. (23) that horizontal spectra have the low-frequency power-law asymptote with the slope $-(\mu-2+v) /(v+1)$. The plots of Figs. 3 and 4 demonstrate vividly that the scale $l_{s}$ may be considered as an outer scale with respect to LI inhomogeneities.

It is interesting to consider the behavior of the 1D oblique spectra at different angles $\vartheta$. They were obtained using Eqs. (4), (18), and (19) for the numerical integra- 


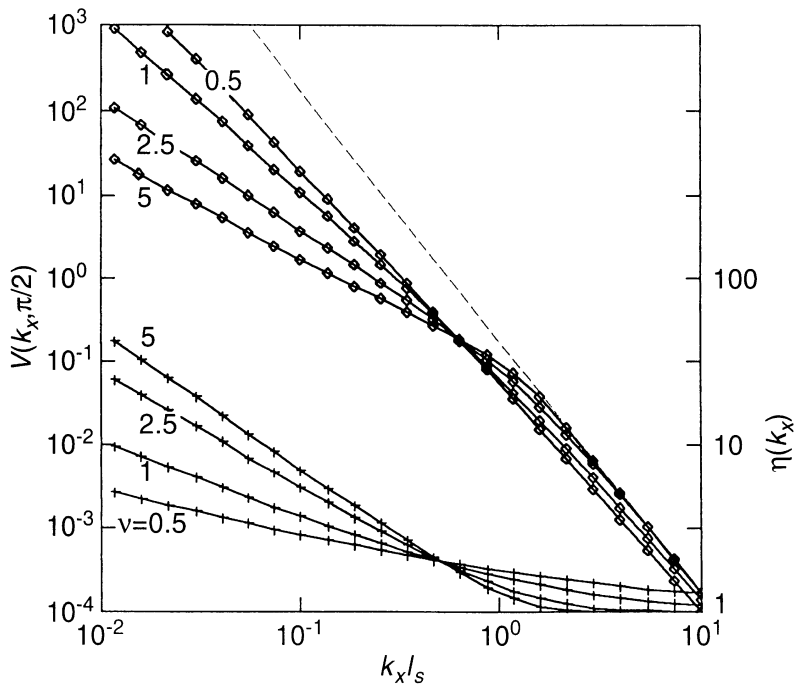

Fig. 4. The 1D horizontal spectra $V\left(k_{x}, \pi / 2\right)$ of LASIs (diamonds) and the 1D spectrum $V_{i s}\left(k_{x}\right)$ of LI inhomogeneities (dashed line) with the same GE spectrum $E(q)=q^{-3} ;$ anisotropy coefficients $\eta=1+\left(q l_{s}\right)^{-v}$ (crosses), $q$ is the solution of the equation $q=k_{x} \eta(q)$; the numbers in the plots are the values of $v$

tion of Eq. (12). Figure 5 shows such spectra for $\mu=5$ and $v=2$. It is seen that at values of $\vartheta 0<\vartheta<\pi / 2$ there is an intermediate asymptote that coincides with the low-frequency asymptote of the horizontal spectrum and has the slope $-5 / 3$ in accordance with Eq. (23). The anisotropy coefficient Eq. (19) increases infinitely at small $q l_{s}$ and means that the temperature field tends to a quasi-layered structure. For this reason the 1D oblique spectrum is determined mainly by a vertical structure at low frequencies during measurements deviating a little from the strictly horizontal direction (Barat and Bertin, 1984). Consequently, the spectral slope of oblique spectra is the same asymptotically at low spatial frequencies as that of vertical spectra.

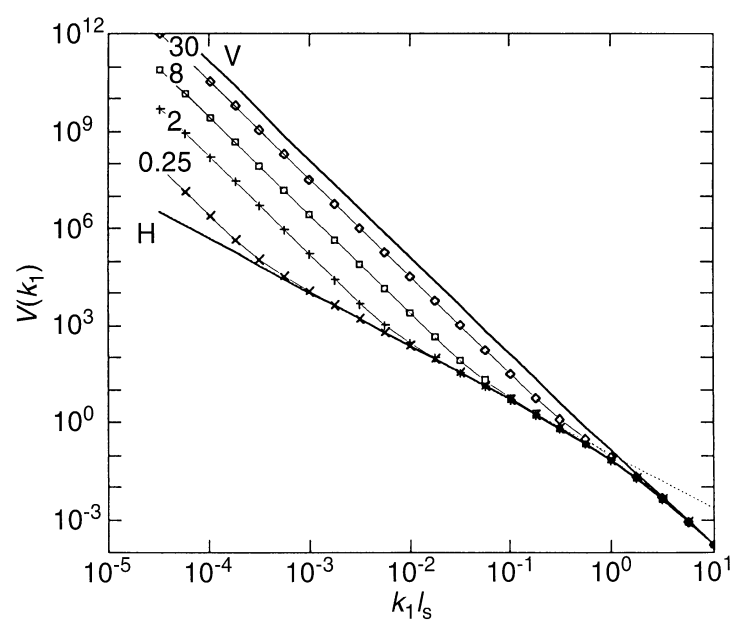

Fig. 5. Oblique 1D spectra at the different values of the angle $\vartheta$ for $\mu=5$ and $v=2$. The numbers by the spectra are $90^{\circ}-\vartheta$, the thick lines correspond to vertical $(\mathrm{V})$ and horizontal $(\mathrm{H})$ 1D spectra, the dotted line is the low-frequency asymptote of the horizontal 1D spectrum
The examples in Figs. 4 and 5 show that the 3D spectrum model with varying anisotropy is able to describe the observed vertical, horizontal, and oblique 1D spectra with different spectral slopes. Therefore the considered model of LASIs may include, for example, the vertical and horizontal temperature spectra suggested in Dewan (1994). Really, as follows from Eqs. (21) and (23), at $\mu=5$ and $v=2$ the vertical and horizontal spectra may have low-frequency parts with slopes -3 and $-5 / 3$ accordingly. If we assume in particular that $S_{T}^{2}$ in Eq. (18) and $l_{s}$ in Eq. (19) are equal to

$S_{T}^{2}=\frac{2 \pi \alpha}{.198}\left(\frac{T(\gamma-1)}{H \gamma}\right)^{2}$ and $l_{s}=1.77\left(\frac{\alpha_{2}}{\alpha_{1}}\right)^{2 / 3} l_{B}$

where $\alpha, \alpha_{1}$, and $\alpha_{2}$ are constants introduced in Dewan (1994), $\gamma$ is the ratio of specific heats, and $H$ is atmospheric scale height, then we obtain from Eqs. (21) and (23) the spectra the same as Dewan's for small wave numbers $\left|k_{z}\right|, k_{h} \ll 1 / l_{s}$. Moreover, if we take into account that $T / H=M g / R$, where $M$ is the molecular weight of air, $g$ is gravity acceleration, and $R$ is gas constant, then we see that the vertical temperature spectra in the model of Dewan (1994) are determined by molecular properties, gravity acceleration, and nondimensional constant $\alpha$, [see also Table 1 in Dewan (1994)]. It is a little surprising because there is no explicit dependence of the vertical temperature spectrum on atmospheric parameters. However, the detailed discussion of the $S_{T}^{2}$ choice is out the scope of the present heuristic model.

\section{Coherency spectra}

It is interesting to consider two-point coherency spectra of temperature fluctuations. Evidently, these spectra should be a more sensitive tool in the study of anisotropy properties as a function of inhomogeneity dimensions. We consider here one example of coherency spectra:

$V\left(k_{x} ; \Delta z, \pi / 2\right)=\int \Phi(k) \cos \left(k_{z} \Delta z\right) d k_{y} d k_{z}$,

where $\Delta z$ is the vertical distance between observation points. It is relatively easy to measure Eq. (25) using a vertically spaced set of sensors moving in the horizontal direction (Dugan, 1984; Marmorino, 1990). To simplify Eq. (25) for the 3D spectrum with the constant anisotropy coefficient $\eta=\eta_{0}$ and Eq. (18), let us introduce the new variables of integration $æ$ and $\varphi$ : $k_{y} \eta_{0} \Delta z=æ \cos \varphi, k_{z} \Delta z=æ \sin \varphi$. We can then calculate the integral on $\varphi$ using the integral representation of Bessel functions and the integral on æ using expression 6.565.4 from Gradshteyn and Ryzhik (1965). The calculation of Eq. (25) for Eq. (18) and at $\eta=$ const. $=\eta_{0}$ shows that the relationship

$\operatorname{Coh}\left(k_{x} ; \Delta z, \pi / 2\right)=\frac{V\left(k_{x} ; \Delta z, \pi / 2\right)}{V\left(k_{x}, \pi / 2\right)}$

is the universal monotone function of $\eta_{0} k_{x} \Delta z$, 
$\operatorname{Coh}\left(k_{x} ; \Delta z, \pi / 2\right)=\frac{(\mu-2)\left(\eta_{0} k_{x} \Delta z\right)^{\frac{\mu}{2}-1}}{2^{\frac{\mu}{2}-1} \Gamma\left(\frac{\mu}{2}\right)} \mathrm{K}_{\frac{\mu}{2}-1}\left(\eta_{0} k_{x} \Delta z\right)$,

where $\mathrm{K}_{\beta}$ is the modified Bessel function. If we take into account the properties of the function $\mathrm{K}_{\beta}$ at small and large arguments we can see that $\operatorname{Coh}\left(k_{x} ; \Delta z, \pi / 2\right)$ tends to 1 at $k_{x} \rightarrow 0$ and tends to 0 at $k_{x} \rightarrow \infty$. The value of $\eta_{0} \Delta z$ is the typical scale of the coherency.

The plots in Fig. 6 show $\operatorname{Coh}\left(k_{x} ; \Delta z, \pi / 2\right)$ as functions of $k_{x} l_{s}$ and $\Delta z / l_{s}$. The numerical calculations of Coh for LASIs were made using Eqs. (18), (19), (25), and (26) at $\mu=5$ and at different $v$ in order to show the dependence on the rate of change of the anisotropy coefficient. The values of $\Delta z / l_{s}=0.5,1,2,4$, and 8 were used for those calculations at $v=2$ and $0.5,1,2,3$, and 4 at $v=16$. The numbers by the curves give these values. Equation (27) was used to calculate $\operatorname{Coh}\left(k_{x} ; \Delta z, \pi / 2\right)$ for LI $\left(\eta_{0}=1\right)$ inhomogeneities with $\mu=5$ and the same $\Delta z / l_{s}$. The curves for LI inhomogeneities move along logarithmic frequency scale and do not change their shape at different values of the parameter $\Delta z / l_{s}$.
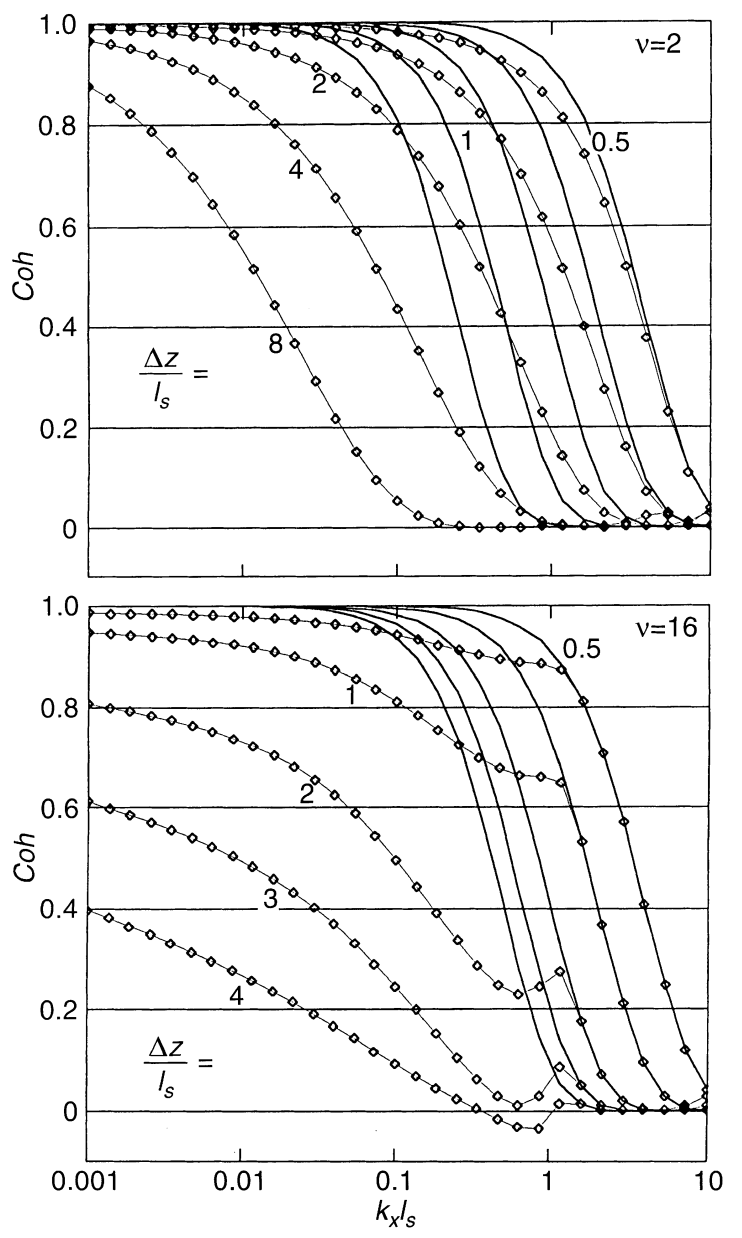

Fig. 6. The coherency spectra $\operatorname{Coh}\left(k_{x} ; \Delta z, \pi / 2\right)$ for $E(q)=q^{-3}$ and $\eta=1+\left(q l_{s}\right)^{-v}: v=2$ (top, diamonds), $v=16$ (bottom, diamonds) and for LI inhomogeneities (thick solid lines); the numbers in the plots are the values of $\Delta z / l_{s}$
Figure 6 shows that $\operatorname{Coh}\left(k_{x} ; \Delta z, \pi / 2\right)$ of LASIs practically coincide at high frequencies $k_{x} l_{s}>1$ with $\operatorname{Coh}\left(k_{x} ; \Delta z, \pi / 2\right)$ of LI inhomogeneities. However, the difference is pronounced at low frequencies $k_{x} l_{s}<1$, especially for large $v$, i.e. for a very sharp change in the anisotropy coefficient. We can see the non-monotonic dependence of $\operatorname{Coh}\left(k_{x} ; \Delta z, \pi / 2\right)$ on $k_{x}$ at $v=16$. The last large value of $v$ corresponds to a very sharp transition from LI to a quasi-layered medium. These results, as well as those shown in Fig. 4 demonstrate the sense of $l_{s}$ as the typical scale of symmetry change.

The coherence spectrum can not be deduced only from knowledge of vertical and horizontal 1D spectra. Therefore Eq. (26) together with Eqs. (15) and (16) may be considered in the general case as the three equations for the two unknown functions $E(q)$ and $\eta(q)$ defining the model, if the results of the $V\left(k_{z}, 0\right), V\left(k_{x}, \pi / 2\right)$, and $\operatorname{Coh}\left(k_{x} ; \Delta z\right)$ measurements are available. This gives the possibility to prove the reliability of estimations of $E$ and $\eta$.

\section{The comparison of the LASIs model with the measurements of both vertical and horizontal temperature spectra}

We will consider the 1D spectra measured by Hostetler and Gardner (1994) as an example of the application of the heuristic model for experimental data parameterization. An analysis of the geophysical meaning of these measurements is not the aim of the present paper. The initial data were obtained using lidars installed aboard an aeroplane. The lidars allowed the measurement of relative perturbations of air density $\Delta \rho / \rho$. Seven pairs of simultaneous measurements of vertical and horizontal spectra of $\Delta \rho / \rho$ are represented in the plots in this paper. In a reasonable approximation, $\Delta \rho / \rho=-\Delta T / T$, where $T$ and $\Delta T$ are mean temperature and its perturbations. We will use the results of the mesospheric measurements.

Both horizontal and vertical spectra have approximately the power-law parts in a rather wide band of spatial frequencies. The slopes of vertical spectra are close to -3 for wave numbers of more than $0.1 \mathrm{~km}^{-1}$. The absolute value of slopes of the horizontal spectra are less. The slope of -3 is in agreement with the different models (Shur, 1962; Lumley, 1964; Van Zandt, 1982; Smith et al., 1987; Sidi et al., 1988; Weinstok, 1990). The stable stratification of the mesosphere allows us to apply the results of Sects. 5 and 6 to the parameterization of measured spectra, if we assume that the hypothesis about local axial symmetry is true for inhomogeneities with vertical sizes less than atmospheric scale height.

It was supposed that the set of experimental data represents the similar physical phenomena. Therefore all measured vertical spectra were put in one plot for analysis by the LASIs model. They occupied a relatively narrow strip on this plot as shown (hatched strip) in Fig. 7a. The situation is the same with the horizontal spectra shown in Fig. 7b. This may be a confirmation of the mentioned supposition. 

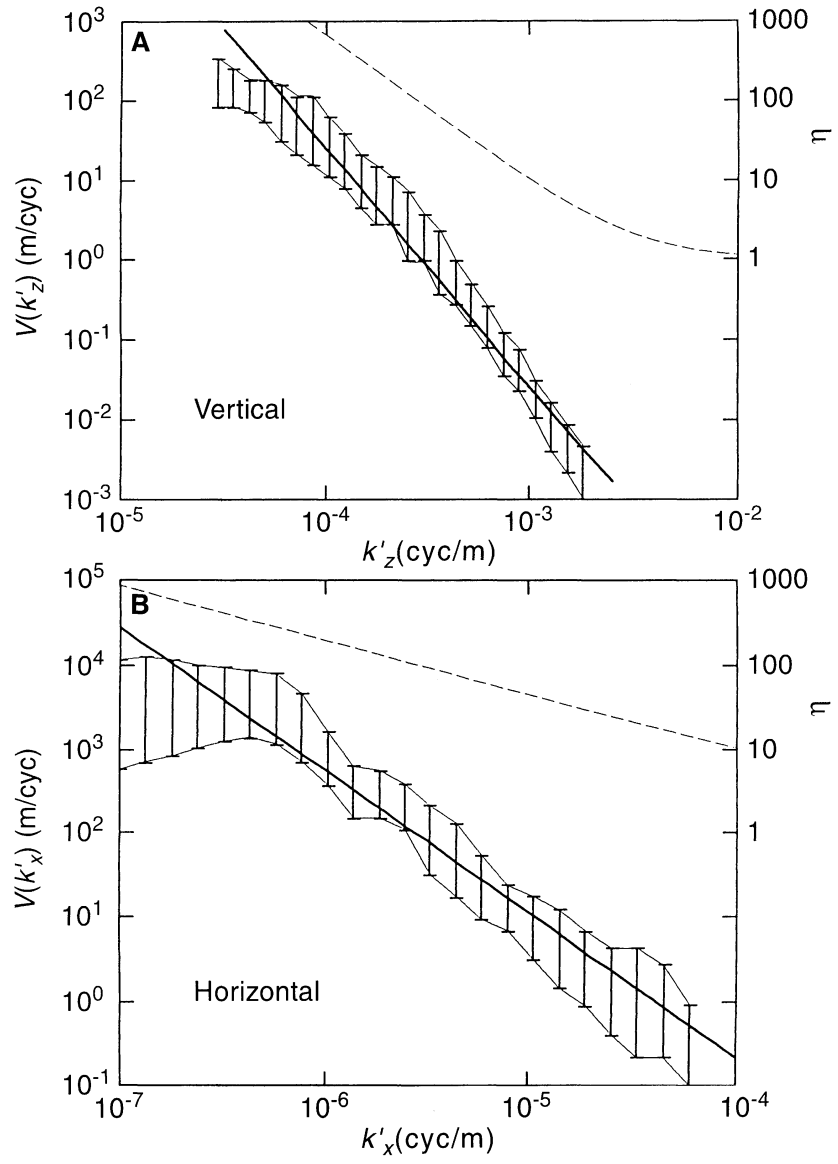

Fig. 7. The comparison of the model (thick, solid lines) with the measurements (Hostetler and Gardner, 1994) (hatched stripes) of vertical $V\left(k_{z}^{\prime}\right)$ (top) and horizontal $V\left(k_{x}^{\prime}\right)$ (bottom) spectra of relative fluctuations of temperature $(T-\langle T\rangle) /\langle T\rangle$ in the mesosphere at altitudes $80 \div 100 \mathrm{~km}$., $k_{z}^{\prime}=k_{z} / 2 \pi, k_{x}^{\prime}=k_{x} / 2 \pi$. The numbers on the right-hand side are the scale for the anisotropy coefficient $\eta$ (dashedlines)

As the experimental 1D spectra have a pronounced power-law part, the following GE spectra $E$ were chosen: $E(q)=S^{2} q^{-\mu+2}$ with $\mu=5$ and with the fitting parameter $S^{2}$. It was assumed also that anisotropy coefficient $\eta$ is defined by Eq. (19) with the fitting parameters $v$ and $l_{s}$.

It is obvious in accordance with the results of Sects. 4 and 5 that a preliminary estimation of the parameter $S^{2}$ may be obtained from vertical spectra. It is possible after this to get a preliminary estimation of the parameters $v$ and $l_{s}$ from the power-law part of the horizontal spectrum using Eq. (23). By using the trial and error method, three parameters wee estimated: $v=1.85, \quad l_{s}=48 \mathrm{~m}$ and $S=3.8 \cdot 10^{-9}(\mathrm{rad} / \mathrm{m})^{2}$. The thick solid lines in Fig. 7a, represent the results of numerical calculation of both vertical and horizontal spectra using Eqs. (15) and (16) and with the stated parameters. The dotted lines in these plots represent the anisotropy coefficient $\eta$.

The plots of Fig. 7 show that the model of LASIs gives the possibility of describing in a single way both 1D spectra - horizontal and vertical - with different slopes. The slope of horizontal spectra is equal to $-(\mu-2+v) /(v+1)=-1.7 \quad$ in accordance with Eq. (23). Note that the $3 \mathrm{D}$ temperature spectrum is defined completely by parameters $S, l_{s}$, and $v$ for $k_{z}^{\prime}>10^{-4} \mathrm{cyc} / \mathrm{m}$ and $k_{h}^{\prime}>10^{-6} \mathrm{cyc} / \mathrm{m}$. A domain of wave number $k_{z}^{\prime}<10^{-4} \mathrm{cyc} / \mathrm{m}$ and $k_{x}^{\prime}<10^{-6} \mathrm{cyc} / \mathrm{m}$ was not considered because the hypothesis of axial symmetry is probably wrong for such large inhomogeneity scales.

The parameterization of vertical and horizontal spectra was carried out in Hostetler and Gardner (1994) on the basis of models in Gardner et al. (1993) and Gardner (1994). The model of Gardner et al. (1993) contains six free parameters. These two models are hardly connected with the linear theory of inner gravity waves. They are based on the ad hoc assumption about the separability of temporal and spatial spectra. These assumptions are not used in the present heuristic model, allowing one also to predict the estimation of typical scale of symmetry change. In order to check this prediction it is necessary to have measurements with better spatial resolution.

\section{The comparison of the LASIs model with the measurements of the coherency spectra of temperature fluctuations}

The equation $\operatorname{Coh}\left(k_{x} ; \Delta z, \pi / 2\right)=$ const. corresponds to the family of isolines on the plane $\left(k_{x}, \Delta z\right)$. If the anisotropy coefficient is $\eta=\eta_{0}$, then, as is seen from Eq. (27), this family is a set of hyperbolae. The numerical solution gives $\eta_{0} k_{x} \Delta z=1.078$ for Eq. (27), $\mu=5$, and const. $=\sqrt{.5}$. The family of the straight lines with slope -1 corresponds to these hyperbolae on a loglog plot. Simple derivations show also that a similar family corresponds to the spectrum by Sidi et al. (1988) in a region $k_{x} \gg k_{*}$ and $k_{*} \Delta z \gg 1$, where $k_{*}$ is the scaling wave number of this spectrum.

The measurements in the stably stratified ocean (Dugan, 1984) showed however that isolines of the coherency spectrum are not straight lines on the log-log plot. It is noted in Dugan's paper that the low-frequency part of experimental data may be described using the Garret-Munk model only. If one smooths his experimental data $\Delta z$ and $k_{x}$ for $\left[\operatorname{Coh}\left(k_{x} ; \Delta Z, \pi / 2\right)\right]^{2}=0.5$ [Fig. 8 in Dugan (1984)] in some reasonable way, it is seen that the curve obtained has a positive curvature. This means that the anisotropy of inhomogeneities changes and that the rate of anisotropy enlargement decreases when inhomogeneity sizes increase. Figure 8 of the present paper shows the experimental data and the family of hyperbolae corresponding to constant anisotropy coefficient.

Therefore, to describe Dugan's results it is necessary to use an equation for $\eta(q)$ a little different to Eq. (19). We assume that the anisotropy coefficient $\eta$ tends to a large value $\eta_{\max } \gg 1$ when $q l_{s}$ tends to zero:

$\eta(q)=1+\frac{1}{\left(q l_{s}\right)^{v}+\eta_{\max }^{-1}}$.

The parameters $l_{s}, \eta_{\max }$, and $v$ were used as fitting parameters to describe the experimental data 


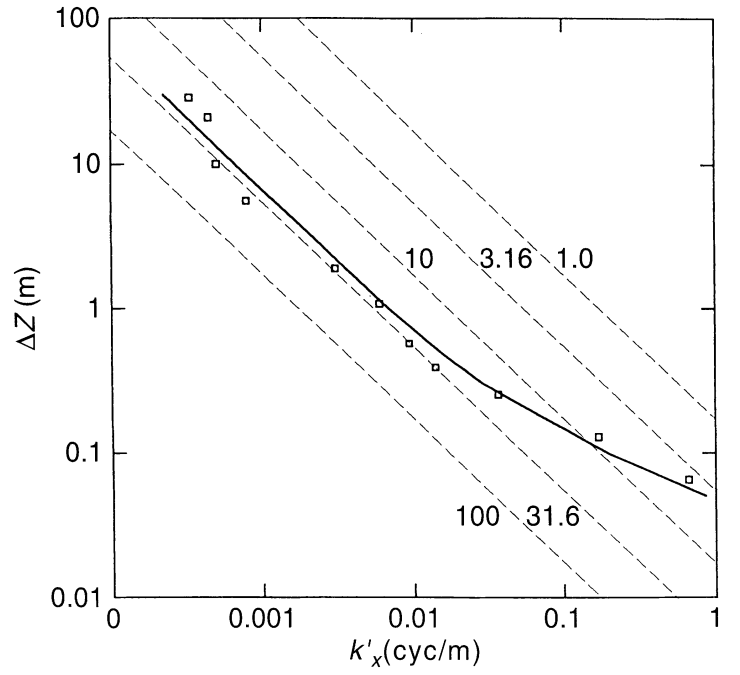

Fig. 8. The comparison of vertical coherence scales $\Delta Z$ : the model (solid line) and the measurements (Dugan, 1984) (boxes). The model dependence $\Delta Z$ on $k_{x}^{\prime}=k_{x} / 2 \pi$ was obtained by the numerical solution of the equation $\left[\operatorname{Coh}\left(k_{x} ; \Delta Z, \pi / 2\right)\right]^{2}=0.5$ and Eqs. (22), (25), (26) with anisotropy coefficient Eq. (28), and GE spectrum Eq. (18). Dashed lines are the hyperbolas $\eta_{0} k_{x} \Delta Z=1.078$ at different $\eta_{0}$, numbers are the values of $\eta_{0}$

$\left[\operatorname{Coh}\left(k_{x}, \Delta Z\right)\right]^{2}=0.5$ using Eqs. (4), (18), (25), (26), and (28). The parameter $\mu$ was chosen as $\mu=5$ as measurements were made under conditions of stable stratification. By using the trial and error method three parameters were estimated: $l_{s}=0.02 \mathrm{~m}, v=2$, and $\eta_{\max }=25$. The numerical analysis showed that the results of the model calculation depend very weakly on values of $v$ and $\mu$. Figure 8 shows the comparison of the experimental data and the model with a variable anisotropy coefficient. The dashed lines correspond to hyperbolae $\eta_{0} k_{x} \Delta Z=1.078$ at different $\eta_{0}=$ $1, \sqrt{10}, \ldots, 100$.

Paremeter $S_{T}^{2}$ is not important in calculations of Coh. However, it was later estimated by the comparison of the model and experimental horizontal spectra of the temperature (Fig. 5 of Dugan's paper). The consequent value of $S_{T}^{2}$ was $S_{T}^{2}=6.3 \cdot 10^{-2} \mathrm{~K}^{2}(\mathrm{cyc} / \mathrm{m})^{2}$. The result of numerical calculation using Eqs. (16), (18), and (28) is shown in Fig. 9. It is possible to see the agreement between the model and experimental spectra. The change of the spectral slope is connected with the saturation of the anisotropy coefficient. Let us not that obtained parameters define completely the 3D temperature spectrum. However, as already stated, the discussion of the geophysical meaning of this spectrum is out the scope of the present paper, in accordance with its initial objectives.

\section{Possible generalization of the developed model}

If we assume that the random field is formed by random propagated monochromatic plane waves with frequencies $\omega$, and that the dispersion equation $\omega=f(\boldsymbol{k})$ is known for these waves, then the simplest applicability

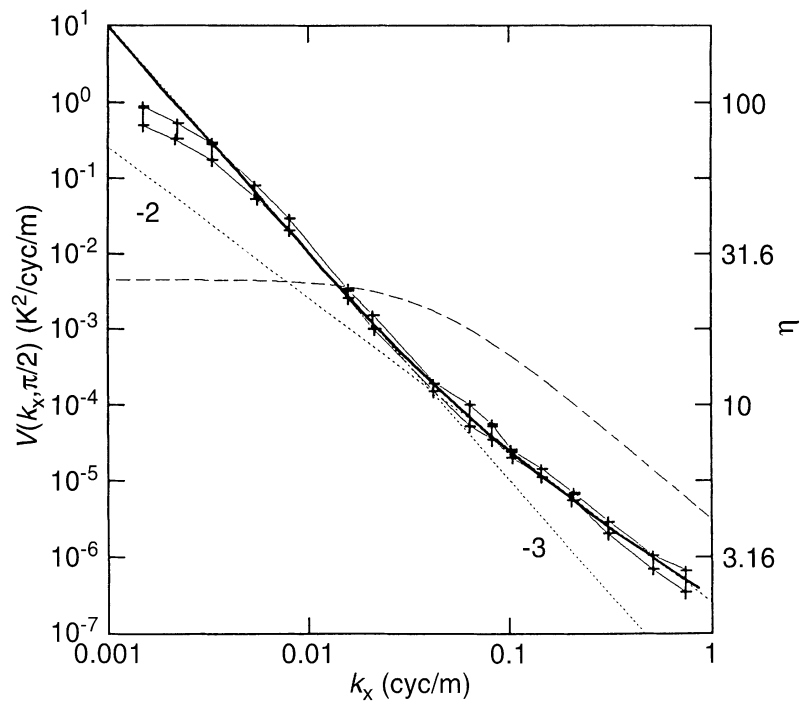

Fig. 9. The comparison of the model horizontal spectrum (thick, solid line) with the measurements by Dugan (1984) (hatched stripe). Dotted lines are the power-law functions with slopes -2 and -3 . The dashed line is the dependence of anisotropy coefficient $\eta$ on $k_{x}^{\prime}$

expansion of the present model consists in the transition to spatial-temporal spectra $\Phi_{s t}(\boldsymbol{k}, \omega)=\Phi(\boldsymbol{k}) \delta(\omega-f(\boldsymbol{k}))$. The dispersion equation $\omega=\boldsymbol{k}\langle\boldsymbol{v}\rangle$ corresponds to the "frozen" turbulence hypothesis. A more complicated case consists in the use of the dispersion equation corresponding to inner gravity waves $\omega^{2}=\left(N^{2} k_{h}^{2}\right.$ $\left.+f^{2} k_{z}^{2}\right) / k^{2}, k^{2}=k_{z}^{2}+k_{h}^{2}, N>\omega>f$.

The following generalization consists in developing the models for the description of such statistical local uniform inhomogeneities of scalar quantity which have the symmetry with respect to three coordinate planes only. This case may correspond, for example to the stratified geophysical flows with shear mean velocity $\langle\boldsymbol{v}\rangle$. The second preferable direction is defined by $\operatorname{rot}\langle\boldsymbol{v}\rangle$. Let us choose the $O y$-axis parallel to $\operatorname{rot}\langle\boldsymbol{v}\rangle$. The $O z$-axis coincides to the vertical. The spectrum of temperature inside such flows is strictly speaking a function of the three independent variables: $k_{x}, k_{y}$, and $k_{z}$. Turbulence in similar flows was studied by Kristensen et al. (1987).

To simplify the description of temperature fluctuations with such a degenerate symmetry, let us introduce the GE spectrum $E(q)$ by assuming that the spectrum $\Phi(\boldsymbol{k})$ has a constant value on a one-parameter family of surfaces. As an example, assume that this is the family of ellipsoids:

$k_{z}^{2}+k_{x}^{2} \eta_{x}^{2}(q)+k_{y}^{2} \eta_{y}^{2}(q)=q^{2}$

whose main axes coincide with the coordinate axes and where $q$ is the parameter of the family as before; $\eta_{x}(q)$ and $\eta_{y}(q)$ are the continuous unambigous functions of $q$. The conditions:

$\frac{q}{\eta_{x}(q)} \frac{d \eta_{x}(q)}{d q}<1 \quad \frac{q}{\eta_{y}(q)} \frac{d \eta_{y}(q)}{d q}<1$

should be fulfilled for all $q>1 / L_{0}$. The conditions of Eq. (30) imply the absence of ellipsoid intersections in Eq. (29). If we assume $\eta \rightarrow 1$ for large $q$ then all wave- 
number space may be filled by ellipsoids of this family under these conditions. Under these assumptions the 3D spectrum $\Phi=\phi(q)$ is a function of one variable $q$. We may now define $E(q)$ as previously:

$E(q)=\frac{4 \pi q^{2}}{\eta_{x}(q) \eta_{x}(q)} \phi(q)\left[1-\frac{q}{3}\left(\frac{d \eta_{x}(q)}{\eta_{x}(q) d q}+\frac{d \eta_{y}(q)}{\eta_{y}(q) d q}\right)\right]$.

To describe the spectrum completely by such approximations, it is necessary to know $E(q)$ and the two functions $\eta_{x}(q)$ and $\eta_{y}(q)$. Thus there exists a possibility to use the three functions of one variable $q$ instead of one function $\Phi(\boldsymbol{k})$ of three variables for such degenerated symmetry. This is probably a more reliable way of approximating experimental data for some cases.

\section{Summary and conclusions}

As is well known, the $3 \mathrm{D}$ spectrum determines all second statistical moments of random fields. Currently, the spectral theory of LI random field is used everywhere. However, the random temperature fields in the stably stratified atmosphere are locally axisymmetric in a wide range of scales. The spectral theory of scalar LAS random fields is a rather complicated. The object of this paper consists in the creation of simple approximate methods of spectral description of such fields independently of their origin. The solution to this problem is based on the generalization of the spectral theory of LI random fields and on the use of symmetry properties.

3D spectrum $F$ of LASIs is a function of two independent variables in a general case. The heuristic spectral model is suggested to simplify the study of LASIs. It is based on the main assumption that the considered 3D spectra are given on the one-parametric family of surfaces of rotation around the axis of symmetry. Such 3D spectra are reduced to a function of one variable, the parameter of the family, $q$. Such an approach allows one to introduce the consideration of the concept of the GE spectrum $E(q)$ which describes a distribution of energy of inhomogeneities, whereas the family of surfaces allows one to describe their shape. The choice of family and GE spectrum provides a flexible and simple tool for the construction of a $3 \mathrm{D}$ spectra model of LASIs.

The family of stretched ellipsoids of rotation is considered in detail as an example of an application of the suggested model. This family is probably the simplest after the family of concentric spheres on which spectra of LI fields are given. The semi-axis of ellipsoids was chosen as the parameter of the family. The ratio of semi-axes $\eta$ is a function of $q$ and $\eta$ characterizes the anisotropy of inhomogeneities quantitatively at each point of wave-number space. The explicit formulae were obtained for $1 \mathrm{D}$ vertical and horizontal spectra.

If the GE spectrum $E(q)$ is the traditional power law then $1 \mathrm{D}$ vertical spectrum depends on anisotropy very weakly and it is very close to a 1D spectrum of LI inhomogeneities with the same GE spectrum. The 1D horizontal spectrum, on the other hand, is sensitive to the anisotropy change. It has two power-law asymptotes with different slopes. The oblique spectra show three asymptotes. Their behavior at low frequencies is a reflection of the vertical structure.

The suggested model of 3D spectra allows one to calculate two-point coherency spectra. The numerical study showed that they are sensitive to the rate of anisotropy coefficient changes.

The examples of experimental data parameterization show the efficiency of the suggested model in an analysis of different measurement results from a unique point of view. The analysis of atmospheric measurements by Hostetler and Gardner (1994) is the first example. It shows that the simplest chosen family of surfaces, the power-law GE spectrum, and the power-law anisotropy coefficient describe the property of vertical and horizontal measured spectra. The theoretical predictions, obtained in the present paper, show that it would be interesting to continue to process the data and to calculate the coherency spectra. They are defined completely by estimated parameters and can be calculated from the existing set of measurements. Consequently the results of the such actions allow one to check additively the estimations of the spectral parameters.

The second example of the application is the analysis of spectral measurements by Dugan (1984) in the stably stratified ocean. The suggested model allows one to explain qualitatively the dependence of the coherency scale on the sizes of the inhomogeneities. This dependence is the consequence of the anisotropy changes. The use of the measured horizontal spectra allows one to estimate all parameters of $3 \mathrm{D}$ spectrum. It also allows the calculation of the vertical spectra which would be interesting to compare with measured vertical spectra, which, in fact, these measurements allow one to estimate. Unfortunately they are not published in Dugan (1984).

Thus these examples show that the suggested heuristic model allows one both to describe quantitatively the different experimental data and to parameterize these in a simple way. The use of this model allows one to plan the effective processing of experimental data. The successful parameterization of the different experimental data suggests that the details of 3D spectra shape are probably not important for the calculation of such integral characteristics as 1D spectra and maybe coherency spectra. This is some justification of the choice of the simplest surface family for the assignment of $3 \mathrm{D}$ spectra. The chosen family of ellipsoids may reflect the main property of axisymmetric inhomogeneities, i.e. the ratio of their vertical and horizontal sizes.

The idea of $3 \mathrm{D}$ spectra representation as functions given on a one-parametric family of surfaces suggests a way of generalizing the model for the analysis of random fields with a more degenerate symmetry than LAS. For example, such a situation arises in the consideration of stratified flows with mean velocity shear. The temperature spectrum in this case may be represented by three functions of one variable instead of one function of three variables. 
The suggested model gives a 3D spectrum, and for this reason it is useful for analysis of radio-wave, light, and sound propagation through the atmosphere. For example, this model may be applied to the study of vertical and horizontal temperature spectra, aspect sensitivity of radar return, and scintillation from a unique point of view (Tatarskii, 1967; Gurvich, 1994).

However, it is necessary to note that this heuristic model is not a consequence of the rigorous theory of fluid mechanics. Consequently, in order to define the GE spectrum and family of surfaces on which a $3 \mathrm{D}$ spectrum is given, it is necessary to use some arguments on the basis of similarity theory, energy balance, and so on, which is usual for most models of temperature spectra. In spite of noted peculiarities, the developed model may be useful in parameterizing and acquiring experimental results, in the planning of measurements and processing of data, and in the discussion of different observed phenomena on the basis of a unique point of view.

Acknowledgements. I acknowledge with pleasure the helpful stimulating discussions with F. Dalaudier and T. E. Van Zandt. The research described in this publication was possible in part by Grant number 96-05-65112 of the Russian Fund of Fundamental Investigations and by Project number 1721 of cooperation CNRS RAN.

Topical Editor D. Alcaydè thanks T. E. Van Zandt and another referee for their help in evaluating this paper.

\section{References}

Alexandrov, A. P., G. M. Grechko, A. S. Gurvich, V. Kan, M. Kh. Manarov, A. I. Pakhomov, Yu. V. Romanenko, S. A. Savchenko, S. I. Serova, and V. G. Titov, Spectra of temperature variations in the stratosphere as indicated by satellite-borne observation of the twinkling of stars (English translation), Izv. Atmos. Ocean. Phys, 26, 1-8, 1990.

Barat, J., and F. Bertin, On the contamination of stratospheric turbulence measurements by the wind shear, J. Atmos. Sci., 41, 819-827, 1984.

Batchelor, G. K., The Theory of Homogeneous Turbulence, Cambridge University Press, Cambridge, 1953.

Chandrasekhar, S., The theory of axisymmetric turbulence, Proc. R. Soc. London, A242, 557-577, 1950.

Dalaudier, F., M. Crochet, and C. Sidi, Direct comparison between in situ and radar measurements of temperature fluctuations spectra: a puzzling result, Radio Sci., 24, 311-324, 1989.

Dewan, E. M., The saturated-cascade model for atmospheric gravity wave spectra and the wave length-period (W-P) relations, Geophys. Res. Let., 21, 817-820, 1994.

Dougherty, J. P., The anisotropy of turbulence at the meteor level, J. Atmos. Terr. Physics, 21, 210-213, 1961.

Doviac, R. J., and D. S. Zrnic, Reflection and scatter formulas for anisotropic turbulent air, Radio Sci., 19, 325-336, 1984.

Dugan, J. P., Towed Observations of Internal Waves and Patches of Fine-Scale Activity, Dynamics of Oceanic Inertial Gravity Waves, Proc. "Aha Hulico'a" Hawaiian Winter Workshop, January 17-20, pp 51-64, University of Hawaii at Manoa, Sponsored by the US Office of Naval Research, and the Department of Oceanography, University of Hawaii, 1984.

Fritts, D. C., and T. E. Van Zandt, Spectral Estimates of Gravity Wave Energy and Momentum Fluxes, Part I: Energy Dissipation, Acceleration, and Constraints, J. Atmos. Sci., 50, 36853694, 1993.

Gardner, Ch. S., Diffusive filtering theory of gravity wave spectra in the atmosphere, J. Geoph. Res., 99, 20601-20622, 1994.
Gardner, Ch. S., Ch. A. Hostetler, and S. J. Franke, Gravity wave models for the horizontal wave number spectra of atmospheric velocity and density fluctuations, J. Geoph. Res., 98, 1035-1049, 1993.

Garret Ch. and W. Munk, Space-time scales of internal waves: a progress report, J. Geoph. Res., 80, 291-297, 1975.

George, W. K., and H. J. Hussein, Locally axisymmetric turbulence, J. Fluid Mech., 233, 1-23, 1991.

Gradshteyn, I. S., and I. M. Ryzhik, Table of Integrals, Series, and Products, Academic Press, New York, London, 1965.

Gurvich, A. S., On the scattering of sound and radio waves by turbulent structures in stratosphere (English translation), Izv. Atmos. Ocean Phys., 30, 1-9, 1994.

Gurvich, A. S., A model of three-dimensional spectrum of locally axisymmetric temperature inhomogeneities in a stably stratified atmosphere (English translation), Izv. Atmos. Ocean. Phys., 31, 196-201, 1995.

Gurvich, A. S., and A. I. Kon, Aspect sensitivity of radar returns from anisotropic turbulent irregularities, J. Electromagn. Waves Appl., 7, 1343-1353, 1993.

Gurvich, A. S., S. V. Zagoruyko, V. Kan, L. I. Popov, V. V. Ryumin, S. A. Savchenko, and P. A. Chochia, Structure of temperature inhomogeneities from observations of atmospheric refraction from the SALYUT-6 Orbital Station (English translation), Dokl. Acad. Sci USSR, Earth Sci. Sect., 259, 27-30, 1983.

Hostetler, Ch. A., and Ch. S. Gardner, Observations of horizontal and vertical wave number spectra of gravity wave motions in the stratosphere and mesosphere over the mid-Pacific, $J$. Geophys Res., 99, 1283-1302, 1994.

Kristensen, L., P. Kirkegaard, and D. H. Lenschow, Squashed Atmospheric Turbulence, Riso National Laboratory, DK-4000, Roskilde, Denmark, 1983.

Kristensen, L., D. H. Lenschow, P. Kirkegaard, and M. Courtney, The spectral velocity tensor for homogeneous boundary-layer turbulence, Boundary-Layer Meterol., 47, 149-193, 1987.

Lumley, J. L., The spectrum of nearly inertial turbulence in a stably stratified fluid, J. Atoms. Sci., 21, 99-102, 1964.

Marmorino, G.O., Turbulent mixing in salt finger stair case, $J$. Geophys. Res., 95, 12983-12994, 1990.

Monin, A. S., On properties of turbulence symmetry in atmospheric boundary layer (English translation), Izv. Atmos. Ocean. Phys., 1, 25-30, 1965

Monin, A. S., Theoretical Geophysical Fluid Dynamics, Evironmental Fluid Mechanics, Vol. 6, Kluwer Academic Publishers, London, New York, 1988.

Monin, A. S., and A. M. Yaglom, Statistical Fluid Mechanics, Vol. 2, MIT Press, Boston, 1975.

Obukhov, A. M., On the energy distribution in the turbulent flow spectrum (in Russian), Izv. AN USSR, Ser. Geograf. Geofiz., 5, 453-466, 1941.

Obukhov, A. M., The influence of hydrostatic forces on the structure of the temperature field in turbulent flow (English translation), Dokl. Acad. Sci. USSR, Earth Sci. Sect., 125, 1246-1248, 1959.

Ozmidov, R. A., On the turbulent exchange in stably stratified ocean (English translation), Izv. Atmos. Ocean. Phys., 1, 853$860,1965$.

Shur, G. N., Experimental investigations of the energy spectrum of atmospheric turbulence, $\mathrm{Tr}$. Tsentr. aerolog. observator., 43, 7990, 1962. (Available through the Aerospace Information Division Library of Congress, AID Report T-63-55.)

Sidi, C., J. Lefrere, F. Dalaudier, and J. Barat, An Improved Atmospheric Buoyancy Wave Spectrum Model, J. Geophys. Res., 93, 774-790, 1988.

Smith, S. A., D. C. Fritts, and T. E. Van Zandt, Evidence of a saturation spectrum of atmospheric gravity waves, J. Atmos. Sci., 44, 1404-1410, 1987.

Sreenivasan, K. R., and R. Narasimha, Rapid distortion of axisymmetric turbulence, J. Fluid Mech., 84, 497-516, 1978.

Tatarskii, V. I., Wave Propagation in Turbulent Atmosphere (in Russian), Nauka, Moscow, 1967. 
Van Zandt, T. E., A universal spectrum of buoyancy waves in the atmosphere, Geophys. Res. Lett., 9, 575-578, 1982.

Weinstok, J., Saturated and unsaturated Spectra of gravity waves and scale-dependent diffusion, J. Atmos. Sci., 47, 2211-2225, 1990.
Woo, R., A. Ishimaru, and F. -Ch. Yang, Radio scintillations during occultations by turbulent planetary atmospheres. Radio Sci., 15, 695-704, 1980. 